Pedro Arturo Martínez, Luis Carlos Paschoarelli, Paula Da Cruz Landim*

\title{
Aproximación acerca de los interrogantes sobre lugar e identidad en el diseño contemporáneo ${ }^{1}$
}

\author{
Approach about questions on place and identity in \\ contemporary design
}

Cómo citar:

Landim, P., Martínez, P., \& Paschoarelli, L. (2018). Aproximación acerca de los interrogante sobre lugar e identidad en el Diseño Contemporáneo. Designia, 5 (2), 67-79.

${ }^{1}$ Este artículo es producto de la revisión documental desarrollada como parte de la investigación "Técnicas tradicionais indígenas para o desenvolvimento de produtos de Design sustentável com Gynerium sagittatum", realizada en el doctorado en Design, Universidade Estadual Paulísta Júlio de Mesquita Filho (UNESP), Bauru, Brasil. Agradecimientos al Departamento Administrativo de Ciencia, Tecnología e Innovación (Colciencias) y al departamento de Sucre (Colombia) por el apoyo otorgado mediante la beca para la formación de capital humano de alto nivel en Sucre, 2014

* Pedro Arturo Martínez. Arquitecto, Universidad Católica de Colombia. Magíster en Educación, Universidad Simón Bolívar. Doctorando UNESP. Profesor de tiempo completo, Corporación Universitaria del Caribe (CECAR).pedro.martinez@cecar.edu.co orcid.org/0000-0002-9024-0918

* Luis Carlos Paschoarelli. Diseñador industrial, UNESP. Investigación postdoctoral en Ergonomía, Universidade de Lisboa (Portugal). Doctorado en Ingeniería de Producción, Universidade Federal de São Carlos (UFSCar). Máster en Proyecto, Arte y Sociedad. Profesor titular del Departamento de Diseño y Docente de Diseño Ergonómico, UNESP. paschoarelli@faac.unesp.br orcid.org/0000-0002-4685-0508 
* Paula Da Cruz Landim

Arquitecta y urbanista, Universidade de São Paulo (USP). Estancia postdoctoral en la Universidad de Arte y Diseño de Helsinki (Finlandia). Doctorado en Arquitectura y Urbanismo, USP. Máster en Geografía, Instituto de Geociencias y Ciencias Exactas, UNESP, campus Rio Claro. Docente de Diseño de producto, profesora del Departamento de Diseño y del postgrado en Diseño, Facultad de Arquitectura, Artes y Comunicación (FAAC), UNESP, campus Bauru. paula@faac.unesp.br orcid.org/0000-0002-1510-7738

\title{
Resumen:
}

En este artículo de revisión se presentan aspectos relevantes sobre cómo la contemporaneidad propone un escenario dinámico en el cual las múltiples tensiones generadas entre los conceptos de lo local-global, identidad-innovación, sentido de lugar y desterritorialización, requieren con urgencia reflexión y reinterpretación en el diseño (Design), la Arquitectura, el Urbanismo y otras disciplinas, con el fin de atender problemáticas de fondo que se viven en la ciudad y el territorio. También se muestra que en la actualidad el Design es un campo de convergencias donde se generan diálogos en la búsqueda de reafirmar el sentido del lugar y la identidad como proceso de diferenciación en medio del mundo globalizado.

\begin{abstract}
:
This review article addresses relevant aspects about how contemporaneity proposes a dynamic reality where multiple tensions generated between concepts such as local -global, identity-innovation, sense of place and deterritorialization urge to be reflected and reinterpreted in Design, Architecture, Urbanism and other disciplines. This is necessary in order to deal with fundamental problems observed in the city and the territory. Also the paper shows Design as a field of convergence where nowadays dialogues are generated in the search for reaffirming sense of place and identity, which represents a differentiation process amidst the globalized world.
\end{abstract}


2Se utiliza "Design" en lugar de "Diseño Industrial" ya que el primer término, de amplia aceptación internacional, hace referencia a la disciplina proyectual orientada a la solución de problemas para el desarrollo de objetos, bienes y servicios. A su vez, recoge una amplia gama de especializaciones, tales como Design visual, de productos, de moda, de interiores, de comunicación y web Design

\section{INTRODUCCIÓN}

La ciudad, como contenedor de relaciones de tipo social, económico, político y simbólico, da cuenta de procesos históricos, culturales y comunicativos en los cuales existen fenómenos expresivos que, a su vez, entran en tensión con procesos de racionalización de la vida social (García Canclini, 1997). La ciudad es un hecho complejo que posibilita diferentes acercamientos a sus dinámicas. Muchas veces, estas chocan con la homogenización de la cultura y las formas de vida en sociedad.

La ciudad contemporánea es un espacio de tensiones y contradicciones (Moura, 2014), un producto de las relaciones establecidas en los sistemas de reorganización mundial conocidos como globalización. En ese escenario es importante la identidad, entendida desde la discusión entre lo local y lo global. Asimismo, es significativo el papel del Design ${ }^{2}$ como mediador en las formas de inclusión y exclusión, o como las llama García Canclini, de "entrada y salida" de la homogenización planteada por realidades económicas, culturales y políticas de la contemporaneidad.

Este artículo presenta algunas manifestaciones de esas tensiones y destaca como el Design aparece ante ellas al cumplir un rol fundamental en su condición de disciplina en permanente reflexión sobre las problemáticas de su tiempo.

\section{LAS REALIDADES DE LA CONTEMPORANEIDAD EN RELACIÓN CON LA IDENTIDAD, LA CIUDAD Y EL DESIGN: LO GLOBAL Y LO LOCAL EN LA PRODUCCIÓN DEL DESIGNCONTEMPORÁNEO}

En el panorama de las cuestiones contemporáneas relativas a identidad, ciudad y Design, sale a la luz la importancia de las tensiones asociadas con la relación centro periferia, global - local, hegemónico - marginal. Esto se debe a ciertas características mundiales que algunos pensadores definen como el sistema-mundo (Wallerstein, 2001), el cual habla sobre desarrollo y globalización, especifica sus lógicas en la actualidad y enmarca distintas sociedades en una sola cultura homogénea, por lo tanto, en una única identidad distintiva (García Canclini, 1995). 
Una contradicción en ese contexto globalizado y homogéneo generado por el sistema-mundo hegemónico es la necesidad cada vez mayor de las culturas nacionales, y por consiguiente, de que las ciudades y los territorios recreen procesos de identidad para generar una mayor visibilidad en el mercado mundial. Al respecto, Rofe y Stein (2011) plantean que se ha venido observando una fuerte y creciente conexión entre disciplinas como el Urbanismo, la Arquitectura, el Design y el marketing urbano para lograr una identidad competitiva de la ciudad:

\section{La globalización continúa exacerbando la competencia interurbana. Esta competencia está impulsando a los gobiernos a buscar formas nuevas y más creativas para desarrollar o mejorar su ventaja competitiva. En este clima, el marketing urbano se convierte en un sitio principal de distinción competitiva. (p. 333).}

Muchos ejercicios sobre el tema de la identidad se han realizado alrededor del mundo: Bangladesh (Banu, 2009), Grecia (Yagou, 2007), Croacia (Vukić, 2009), Australia (Worden, 2009; Rofe \& Stein, 2011), India (Kalyan, 2011); y en Latinoamérica, Henriques, Lima, Plácido, Dos Santos y Paschoarelli (2014) en Brasil y Pacheco, Barrero y Gómez (2013) en Colombia, entre otros. Los casos se registran especialmente en lugares considerados en vías de desarrollo o marginales, en una búsqueda por entrar de manera competitiva en el mercado mundial.

En estos ejercicios el sentido de lugar en la ciudad y su relación con el Design son elementos importantes. En la gran mayoría de ciudades se ha verificado una homogenización occidental que ha llevado a la destrucción de las identidades previas para dar paso a una hibridación cultural. En la contemporaneidad, paradójicamente, esta hibridación genera de nuevo procesos de valorización de la identidad local de la mano del Design. Sobre este particular, Kalyan (2011) describe la fragmentación de la identidad local urbana en la India como resultado de los intereses homogenizantes de la globalización:

El urbanismo neoliberal fragmenta las ciudades internamente, excluyendo a los participantes que no son parte de esta nueva experiencia económica a través de la securitización y vigilancia social de nuevos espacios económicos que son un verdadero escape del entorno social inmediato. El urbanismo neoliberal se basa en el surgimiento de la arquitectura "paranoica" o carcelaria basada en la protección, vigilancia y exclusión, y finalmente, en la creciente presencia de la simulación dentro de los paisajes urbanos, imaginaciones de ciudades alternativas a la "terrible realidad" de ciudades reales, por ejemplo, parques temáticos, centros comerciales temáticos con vigilancia policial y formas más sutiles de simulación que invaden la vida cotidiana. (p. 41). 
Desde los planteamientos de Kalyan (2011), es posible entender como el paisaje urbano paulatinamente se va transformando a partir de intereses económicos y políticos para generar nuevas experiencias urbanas en la ciudad contemporánea. Ahora bien, en la India y en otros países, el Design aparece como un instrumento mediador y generador de diálogos que van más allá de los desplazamientos y las exclusiones del pasado para encontrar sentido a los problemas del presente.

$\mathrm{Al}$ analizar varios referentes es posible encontrar interrogantes al interior del Design -como un amplio campo de convergencia y reunión de múltiples disciplinas- que definen dos posiciones contradictorias enmarcadas en los procesos de hibridación contemporánea. Por una parte, la ruptura con el pasado y la generación de nuevas identidades en una búsqueda de innovación; por otra, la intención de establecer contactos con el pasado y estimular la apropiación, las identidades regionales y locales para reafirmar el sentido del lugar como distinción en el mercado global.

La idea de romper con el pasado y producir nuevas identidades viene de la mano con argumentos que identifican como problema central la inseguridad y falta de confianza de los nuevos diseñadores en la búsqueda por crear lenguajes acordes con las necesidades de hoy. Esto puede observarse en lo planteado por Yagou (2007) al describir el debate sobre identidad y Design contemporáneo en Grecia:

Los sentimientos de inseguridad respecto al subdesarrollo institucional pueden tomar formas diferentes. En Grecia, estos sentimientos han sido expresados principalmente por una búsqueda de larga data, insistente y a menudo desesperada por la identidad nacional (...) Se ha argumentado que esta crisis de identidad constituye la cuestión central de la sociedad neohelénica y el eje principal alrededor del cual se desarrolló la historia griega moderna (p. 145). 
Yagou (2007) critica que los diseñadores carecen de confianza para crear, sin basarse en clichés y fórmulas preconcebidas de una identidad nacional, en este caso la griega, productos dirigidos a las necesidades actuales. Aquí se piensa la identidad como un elemento restrictivo que relega problemas contemporáneos.

A través de la crítica al uso de materiales locales, la identidad también es vista como restrictiva para la innovación y el diseño. Por ejemplo, Kalyan muestra en palabras de arquitectos locales de la India que lo local puede ser un obstáculo al desarrollo:

\begin{abstract}
La arquitectura vernácula que usa materiales locales está muerta. El uso de materiales locales se ha vuelto más caro. Hay materiales procedentes de China que resultan más baratos que los materiales locales. La forma más honesta de construcción en cualquier punto de la historia ha sido la más barata, la más rápida y la más superior. Hoy, si las opciones globales proporcionan esas cualidades, obviamente las optaré (2011, p. 45).
\end{abstract}

Se describe aquí la pertinencia de abordar problemas contemporáneos como la inmediatez, la economía y la eficiencia, a los que el diseñador debe responder desde la Arquitectura como una responsabilidad histórica.

La contraparte a estos planteamientos se refleja en la idea de entender la identidad como un camino para potencializar la innovación y diferenciación de productos en aras de abarcar cada vez más necesidades en un mercado altamente competitivo. Un ejemplo de esta visión es el desarrollo y afirmación del Design brasilero que desde la segunda mitad del siglo XX se ha abierto camino hacia la diferenciación en el mercado mundial a través del reconocimiento de lo local. Particularmente, fue en la década de los ochenta cuando los diseñadores comenzaron a reflexionar y discutir sobre problemas de identidad en la producción nacional (De Castro, 2007).

En palabras de Ota (2012), la identidad en el Design brasilero trasciende hacia los objetos. Memorias y herencias culturales actúan en el proceso creativo para lograr la diferenciación en el mercado globalizado: “Una lectura del Design brasileño es un gran viaje. Ejercitamos la memoria histórica, inventamos el universo de nuestra cultura material. Rica en herencias culturales de las más diversas, los objetos hablan de nuestras vidas, nos hacen entender y revisar nuestro pasado (p. 23). 
Trabajos como el de Henriques (2010) y Henriques et al. (2014), evidencian una inquietud por relacionar aspectos de identidad local de las ciudades y reflexionar sobre las marcas urbanas, más allá de aquellas causadas por las administraciones gubernamentales. Mediante un estudio comparativo, Henriques contrasta la búsqueda de identidad en ciudades españolas con inquietudes similares en Brasil. Así, identifica que si bien el concepto de país es diverso y abstracto, las marcas adquieren sentido y valor al ser usadas como representación de la ciudad y del país.

Igualmente, se han realizado ejercicios que buscan reinterpretar la identidad por medio de procesos cada vez más frecuentes en la práctica del Design contemporáneo, como son la revalorización del artesanado y las técnicas tradicionales. Entre otros ejemplos, se encuentran los trabajos de Yair, Press y Tomes (2001), Tung (2012), Pacheco, Barrero y Gómez (2013), Campbell (2014), Alexandre, Gomez y Valente (2015), como también los reunidos en el documento publicado por la Organización de las Naciones Unidas para la Educación, la Ciencia y la Cultura (Unesco, 2005), en los que se exponen posibilidades de transformación a través de iniciativas orientadas a resignificar lo local de la mano del Design.

\section{DISCUSIÓN}

Las diversas posiciones y acciones observadas en torno a la relación identidad, lugar y Design en la contemporaneidad conllevan a una discusión sobre estos temas. A partir del panorama en tensión y el contexto problemático que se ha identificado, resulta interesante y clave para el análisis retomar el interrogante planteado por Hall y Du Gay (2003): ¿quién necesita la identidad?

En la misma dirección también es posible preguntar: ¿la ciudad necesita identidad? Una respuesta rápida puede ser que todos los territorios y grupos sociales precisan de identidad, la cual ayuda a definirlos y diferenciarlos. No obstante, en el mundo de hoy, en constante movimiento, las respuestas son necesariamente mucho más complejas, al ser difícil construir identidad sobre algo en continua transformación. 
Cardoso (2012) se refiere al dinamismo de la identidad al afirmar que esta "siempre es compuesta, construida a partir de muchas partes y configurada por muchas facetas" (p. 91). En este sentido, el dinamismo es igualmente inherente a la identidad en la ciudad, no como algo estático sino como un hecho en permanente construcción. Tanto así que en la construcción y el desarrollo de la imagen de la ciudad contemporánea, la globalización ha estandarizado la imagen de las grandes urbes y ha dificultado diferenciar dónde se interactúa, dada la similitud de códigos y mensajes distribuidos a través de los canales transnacionalizados de comunicación.

La ciudad en busca de redefinición necesita con urgencia procesos de diferenciación que la posicionen nuevamente en el mercado internacional. Esto es posible a través de una reflexión sobre la identidad y el sentido de lugar de cada territorio. Así se requiere sobre todo en la periferia, en los llamados países del tercer mundo, donde los mensajes y códigos llegan distorsionados por las relaciones de poder que imponen ideales del deber ser y modelos a seguir.

Otro hecho de interés para la discusión es la construcción de identidad por parte de las disciplinas involucradas en el proceso de diseño como mediadoras y gestoras de comunicación en la ciudad y el territorio. ¿Cómo reflexionan sobre identidad para generar productos innovadores que den sentido de lugar y representen una marca de origen? Esta reflexión es relevante si bien para algunos (Yagou, 2007) conlleva al problema de las limitaciones para la creación de formas y nuevos productos.

Al respecto, Cardoso (2012) propone la memoria como un elemento clave para los diseñadores en la producción de objetos innovadores y con una fuerte carga de significados que propicie reflexiones sobre identidad y sentido de lugar:

La identidad está en flujo constante, sujeta a la transformación, equivale a una suma de experiencias, multiplicadas por las inclinaciones y divididas por las memorias (...) los buenos diseñadores hacen uso estratégico de la memoria para refinar sus proyectos, introduciéndoles capas adicionales de significado. (p. 92). 
Para Cardoso, la memoria no es un contenedor estático sino un instrumento de autorreflexión que potencia múltiples opciones de significación en el objeto y de aprehensión por parte del usuario. No se entiende como un elemento restrictivo sino, por el contrario, dinámico y propositivo, un posibilitador de la innovación.

Manzini (2009), por su parte, explora la necesidad de un nuevo conocimiento del Design en relación con las maneras contemporáneas de producir y la problemática de un desarrollo local en la sociedad global. Manzini (2014) destaca una "cultura del Design", muy promisoria en un mundo cada vez más conectado, al igual que "Design as place maker" (p .98), concerniente al énfasis del Design en la regeneración de lo local a través de la creación de una nueva ecología del lugar, es decir, de un ecosistema con relaciones armónicas entre la cultura propia y la producción

Huybrechts, Dreessen, Schepers y Calderon (2016), entienden esa aproximación desde los diálogos democráticos facilitados por el Design para direccionar cambios estratégicos que en la ciudad contemporánea se materializan "vía artefactos, como sujetos o escenarios que hacen posibles otras experiencias" (p. 101). Tales diálogos son urgentes, en vista de la desintegración resultante de las problemáticas sociales.

Otra inquietud para la reflexión es: ¿cómo puede el Design atender aspectos de identidad y valores profundos de los grupos humanos, y al mismo tiempo acciones de marketing de la ciudad? Es preciso considerar que estas últimas responden a intereses económicos trasnacionales, por lo general desligados de los valores sociales y culturales de determinados colectivos. Bonsiepe (2011) describe esta problemática desde el campo del Design en los siguientes términos:

\section{El Design se transformó en evento mediático, en espectáculo acompañado por un número respetable de revistas que funcionan como cajas de resonancia para ese fin. Hasta los centros de promoción del Design se encuentran expuestos a esa complicidad de los vehículos de comunicación, corren el riesgo de desvirtuar su objetivo de difundir Design como resolución inteligente de problemas, y no sólo estilización. (p. 18).}

Bonsiepe critica así algunas posiciones del Design contemporáneo, preocupadas más por fines comerciales y de marketing que por resolver problemáticas sociales de fondo. En dichas condiciones, el diseñador actúa posiblemente subordinado a directrices cuyos intereses son la "privatización estética" del rating, en palabras de García Canclini (1997, p. 48), al examinar la subordinación de la cultura latinoamericana a los grandes monopolios. Bonsiepe apunta a entender los procesos de Design e identidad para las ciudades y los territorios actuales como una oportunidad, particularmente en los países periféricos que pueden reafirmar así valores amenazados por la competitividad y el libre mercado del mundo globalizado. 
Costa (2014) dirige la atención hacia esos procesos en que el Design, como disciplina, está llamado a atender problemáticas complejas de la contemporaneidad. Para trabajar acertadamente en este contexto dinámico es necesario romper barreras disciplinarias y entablar diálogos que ofrezcan alternativas en un mundo con múltiples intereses en juego: “Una profesión no se hace solamente recortando bien su área, sino creando vallas e intersecciones que favorezcan la cooperación transdisciplinaria presente en la sociedad y que se nos pide profesionalmente" (p. 67). Se requiere entonces la participación del Design junto a la Arquitectura, el Urbanismo y las artes para analizar los problemas desde distintas perspectivas.

Lo que en algunos casos se entiende como demandas provenientes de intereses trastocados puede ser convertido en oportunidades de acción y trasformación social que beneficien grupos humanos tradicionalmente relegados e invisibilizados.

\section{CONCLUSIONES}

Es posible formular conclusiones preliminares en el camino de enfocar búsquedas profundas para resolver las preguntas relacionadas con las cuestiones sobre identidad, ciudad y Design planteadas en el panorama contemporáneo identificado.

La contemporaneidad propone un escenario dinámico en que las tensiones entre conceptos como local - global, identidad - innovación, desterritorialización - sentido de lugar, precisan cada vez con mayor urgencia de reflexión y reinterpretación. Design, Arquitectura, artes y otras disciplinas deben generar diálogos productivos para afrontar problemas de fondo propios de los flujos y cambios del mundo de hoy.

Son variadas las posiciones respecto a la relación entre los conceptos de ciudad e identidad, al igual que los análisis y cuestionamientos sobre el particular propuestos desde del Design. Lo cierto es que si bien existen posturas contradictorias en cuanto al vínculo identidad - sentido de lugar en el diseño contemporáneo, las reflexiones en torno al tema en el panorama de la contemporaneidad exigen gran atención.

La memoria, la cultura, la identidad y la contextualización temporal siguen teniendo vigencia en los procesos de innovación exigidos por el mundo de hoy. Se trata de valores fundamentales para examinar las posibilidades de diferenciación en un mercado cada vez más competitivo en el que tanto los objetos como la ciudad y el territorio han de ser repensados para responder a las necesidades existentes. 


\section{REFERENCIAS BIBLIOGRÁFICAS}

Alexandre, C. B., Gomez, E. A. \& Valente, A. C. (2015). Interdisciplinary relationship between Designer and Craftsman based on Integrated Craft Manufacturing Systems. Procedia Engineering, 132, 1089-1095.

Banu, L. (2009). Defining the Design Deficit in Bangladesh. Journal of Design History, 22(4), 309-323.

Bonsiepe, G. (2011). Design, cultura e sociedade. São Paulo: Blucher.

Campbell, W. (2014). Designs: An Archaeology of "Craft” as God Term. Computers and Composition, 33, 50-67.

Cardoso, R. (2012). Design para um mundo complexo. São Paulo: Cosac Naify.

Costa, C. Z. (2014). Novas demandas para o design brasileiro contemporâneo. En M. Moura (Comp.). Design Brasileiro contemporâneo: reflexões (pp. 47-67). São Paulo: Estação das Letras e Cores.

De Castro, J. (2007). Design com identidade: por meio de estudos sócio-culturais e dos signos. Tesis de Maestría, Universidade Estadual Paulista, Bauru, SP, Brasil. Disponible en: http://www.faac.unesp.br/\#!/ pos-graduacao/mestrado-e-doutorado/design/dissertacoes--teses/

García Canclini, N. (1997). Imaginarios urbanos. Buenos Aires: Editorial Universitaria de Buenos Aires.

García Canclini, N. (1995). Consumidores y ciudadanos: conflictos multiculturales de la globalización. México: Grijalbo.

Hall, S. \& Du Gay, P. (Eds.). (2003). Questions of cultural identity. Londres: Sage. 
Henriques, F. (2010). Lugares à venda: a construção de um processo visual a partir das marcas- território. Tesis de Doctorado, Pontifícia Universidade Católica de São Paulo, Brasil.

Henriques, F., Lima, A., Plácido, J. C., Dos Santos, M. \& Paschoarelli, L. (2014). Marcas e lugares: um estudo sobre marcas turísticas no Rio de Janeiro. Revista Brasileira de Expressão Gráfica, 2(1), 101-116.

Huybrechts, L., Dreessen, K., Schepers, S. \& Calderon, P. (2016). Democratic dialogues that make cities 'work'. Strategic Design Research Journal, 9(2), 100-111. DOI: 10.4013/sdrj.2016.92.05

Kalyan, R. (2011). Fragmentation by Design: Architecture, Finance, and Identity. Grey Room, 44, $26-53$.

Manzini, E. (2014). Design in a changing, connected world. Strategic Design Research Journal, 7(2), 95-99. DOI: $10.4013 /$ sdrj.2014.72.06

Manzini, E. (2009, enero). New design knowledge. Design Studies, 30(1), 4-12.

Moura, M. (Comp.). (2014). Design Brasileiro contemporâneo: reflexões. São Paulo: Estação das Letras e Cores.

Organización de las Naciones Unidas para la Educación, la Ciencia y la Cultura. (2005). Designers meet artisans. A practical guide. New Delhi, India: Craft Revival Trust - Artesanías de Colombia - Unesco. Disponible en: http://unesdoc.unesco.org/images/0014/001471/147132EO.pdf

Ota, M. (2012). Vanguarda sempre. En R. De Faria (Ed.). Um olhar sobre o design brasileiro (pp. 22-27). São Paulo: SENAI-SP. Disponible en: https://books.google.com.br

Pacheco, J., Barrero, G. \& Gómez, G. (2013). An Eco-Technological Approach to Handcraft Production. Two Cases in the Colombian Caribbean Region. Cuadernos de Desarrollo Rural, 10(70), 115-129.

Rofe, M. \& Stein, L. (2011, agosto). Shedding New Light on Adelaide? Intersections between Urban Design Projects and City Marketing. Journal of Urban Design, 16(3), 333-350. 
Tung, F. W. (2012). Weaving with Rush: Exploring Craft-Design Collaborations in Revitalizing a Local Craft. International Journal of Design, 6(3), 71-84.

Vukić, F. (2009). Hybrid Identities and Paralyzing Traditions: Contemporary Croatian Design within the Context of Social Transition. Design Issues, 25(4), 80-90.

Wallerstein, I. (2001). El eurocentrismo y sus avatares: los dilemas de las ciencias sociales. Revista de Sociología, 15, 27-39.

Worden, S. (2009). Aluminium and Contemporary Australian Design: Materials History, Cultural and National Identity. Journal of Design History, 22(2), 151-171.

Yagou, A. (2007). Metamorphoses of Formalism: National Identity as a Recurrent Theme of Design in Greece. Journal of Design History, 20(2), 14-159.

Yair, K., Press, M. \& Tomes, A. (2001, julio). Crafting competitive advantage: crafts knowledge as a strategic resource. Design Studies, 22(4), 377-394. 